\title{
Acute effects of electronic cigarette smoking on ventricular repolarization in adults
}

\author{
Vahit Demir ${ }^{1}$, Siho Hidayet ${ }^{2}$, Yaşar Turan ${ }^{1}$, Hüseyin Ede ${ }^{1}$
}

1. Department of Cardiology, Medical Faculty of Bozok University,Yozgat, Turkey

2. Department of Cardiology, Medical Faculty of Inonu University, Malatya, Turkey

Siho Hidayet, shhidayet@hotmail.com, GSM: +904223770100; Yaşar Turan, yasar044@hotmail.com, GSM: +903542127060; Hüseyin Ede, huseyinede@gmail.com, GSM: +97450630593

\begin{abstract}
Background: Electronic cigarette (e-cigarette) use is constantly increasing. However, the association between e-cigarette use and ventricular arrhythmia is unknown. Thus, in this study, we aimed to evaluate the markers of ventricular repolarization such as QT interval, corrected QT (QTc), QT dispersion (QTd), peak-to-end interval of the T wave (Tp-e), corrected Tp-e and $\mathrm{Tp}$-e/QT ratios in e-cigarette users.

Methods: The study population consisted 36 e-cigarette users and 40 healthy subjects. Ventricular repolarization parameters were obtained from 12-lead resting electrocardiogram. Ventricular repolarization parameters of the groups were compared. Results: Basal demographic and laboratory data were similar in both groups. According to the electrocardiographic parameters, the Tp-e interval, corrected $\mathrm{Tp}$-e, and Tp-e/QT ratio were significantly higher in individuals using e-cigarettes than in control subjects [74.9 \pm 6.4 milliseconds (ms) vs. $80.1 \pm 4.1 \mathrm{~ms},<0.001 ; 82.9 \pm 7.5 \mathrm{~ms}$ vs. $87.8 \pm 6.3 \mathrm{~ms}, \mathrm{p}=0.003$; $0.20 \pm 0.01$ vs. $0.21 \pm 0.01, \mathrm{p}=0.002$; respectively].

Conclusion: This is the first study to show the disruption of ventricular repolarization properties in e-cigarette users. E-cigarette use in terms of public health leads to augmentation of transmural dispersion of repolarization, which may be potential indicator of ventricular arrhythmogenesis.
\end{abstract}

Keywords: Electronic cigarette; ventricular repolarization; Tp-e interval; Tp-e/QT ratio.

DOI: https://dx.doi.org/10.4314/ahs.v20i4.33

Cite as: Demir V, Hidayet S, Turan Y, Ede H. Acute effects of electronic cigarette smoking on ventricular repolarization in adults. Afri Health Sci. 2020;20(4):1793-9. https:// dx.doi.org/10.4314/ahs.v20i4.33

\section{Introduction}

The effects of conventional cigarette on cardiovascular system have been extensively studied with numerous studies in adults. It is known that smoking is one of the main causes of cardiovascular events and causes ventricular arrhythmias and sudden cardiac deaths ${ }^{1}$. In recent years, electronic cigarettes (e-cigarette) have been launched as an auxiliary method for cessation of smoking by being claimed to be less harmful. Therefore, the frequency of e-cigarette use demonstrated a serious

\footnotetext{
Corresponding author:

Vahit Demir,

Department of Cardiology, Medical Faculty

of Bozok University

Adress: Çapanoğlu Mah. Cemil Çiçek Cad Bozok

Üniversitesi Erdoğan Akdağ Yerleşkesi Atatürk

Yolu 7. KM, 66100 Azizli/Yozgat

GSM: 0090507231 8182,

Fax: +90 (354) 2171072

E-mail: dr.vdemir@hotmail.com
}

increase every year. E-cigarette is a source of nicotine designed as a pen or an accessory that gives the sense of smoking with an appearance similar to conventional cigarette ${ }^{2}$. Although e-cigarette is thought to be less harmful than normal cigarette, such thought does not implicate the safety of e-cigarette use $\mathrm{e}^{3,4}$.

Electrocardiography (ECG) parameters have been recommended to be used in cardiovascular risk screening independently from conventional cardiovascular risk factors in middle age group 5 . Various methods such as ventricular repolarization, QT interval, QT dispersion (QTd) and transmural dispersion of repolarization have been used for this purpose. Regional QT interval changes measured on ECG reflect the altered regional ventricular repolarization. Although the difference of maximum and minimum QT interval between derivations has been defined as QT dispersion (QTd), this may not directly reflect the dispersion of repolarization. Because QTd can be affected by the changes in T-wave shape and errors in QT interval measurements ${ }^{6}$. Abnormalities in ventricular repolarization increases sensitivity to ventricular arrhythmias. Recent studies stated

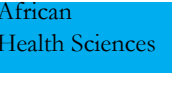

1793

(C) 2020 Demir V et al. Licensee African Health Sciences. This is an Open Access article distributed under the terms of the Creative commons Attribution License (https://creativecommons.org/licenses/BY/4.0), which permits unrestricted use, distribution, and reproduction in any medium, provided the original work is properly cited. 
that the distance ( $\mathrm{Tp}$-e) between the part where T-waves peak (p) and ends (e), as measured in surface ECG, may better reflect the global dispersion of repolarization ${ }^{7}$. Increased Tp-e interval may be a useful marker to demonstrate ventricular arrhythmia and cardiovascular mortality. Tp-e interval is affected by the changes in the heart rate. Therefore, Tpe/QT ratio is a better indicator of ventricular repolarization ${ }^{7}$. QT time, Tp-e interval, Tp-e/QT ratio are ECG variables with demonstrated importance in the estimation of arrhythmia and mortality development in various heart diseases ${ }^{8-11}$.

There is a lack of data on the long-term effects of e-cigarettes on the cardiovascular system. However, in studies examining the acute effects of e-cigarettes, it has been shown that e-cigarette causes potential cardiovascular toxicity in relation to increased heart rate, blood pressure and systemic oxidative stress ${ }^{4}$. This has led to the necessity for investigating the adverse potential effects of e-cigarette use on cardiovascular health outcomes. There is no study in the literature that examines the effects of e-cigarette on the parameters of ventricular repolarization.

The purpose of this study was to evaluate the acute effects of e-cigarette use on ventricular repolarization parameters [QT interval, corrected QT (QTc), QT dispersion (QTd), peak-to-end interval of the $\mathrm{T}$ wave ( $\mathrm{Tp}$ e), corrected Tp-e and ve Tp-e/QT ratios]

\section{Material and Method}

This cross-sectional, prospective study was carried out at the Department of Cardiology, Yozgat Bozok University Faculty of Medicine, Turkey. Between June 2017 and June 2019, 36 e-cigarette users who were followed up at the cardiology outpatient clinic and 40 healthy subjects with similar age and sex and did not use any tobacco and nicotine-containing products were included in the study (76 individuals in total). Subjects aged between 18 and 55 years old who did not have any accompanying diseases, did not smoke conventional cigarettes and had been using e-cigarette for at least 6 months formed the e-cigarette group.

Individuals who consumed caffeine-containing beverages or stimulating substances within 12 hours prior to ECG, alcohol users (>112 gram/week ethyl alcohol intake in men, $>72$ gram/week in women), patients with acute infections, chronic inflammatory disease, diabetes mellitus or hypertension, patients with diagnosed coronary arteri disease (those with ischemia proof in stress test or $>50 \%$ stenosis in any main vessel during coronary angiography or those with history of coronary revascularization), patients with dysrhythmia (atrial fibrillation, sustained atrial arrhythmia, those with $>500$ premature ventricular complex/24 hour in Holter or presence of a PVC on index resting ECG), electrolyte imbalance (serum potassium $<4 \mathrm{mEg} / \mathrm{L}$ or serum magnesium $<0.8 \mathrm{mmol} / \mathrm{L}$ ) and patients whose ventricular repolarization parameters couldn't be calculated due to technical reasons were excluded from the study. Patients with a history of smoking in the last six months were excluded from the study.

Variable mode third generation e-cigarette users were assigned to the e-cigarette group. In the e-cigarette group, e-liquid kits containing medium to high density nicotine $(16-21 \mathrm{mg} / \mathrm{ml})$ were used for the device. Separate settings were used for e-cigarette users. Subjects were recommended to use the e-cigarette for a total of 25 puffs and 5 minutes in accordance with the manufacturer's instructions for use. Again in accordance with the manufacturer's recommendations, each breath was recommended to last for approximately $2-3$ seconds. In accordance with the manufacturer's instructions, it was recommended that the battery be fully charged before use.

As the purpose of the study was to examine the acute effects of e-cigarette exposure on ventricular repolarization, standard 12-lead surface electrocardiography of the participants were obtained by using Nihon Kohden, Tokyo, Japan ECG device within 10 minutes following the use of nicotine-containing e-cigarette. Body mass index (BMI) was calculated by dividing body weight by the square of the neck $\left(\mathrm{kg} / \mathrm{m}^{2}\right)$. Transthoracic echocardiographic examination was performed with Philips Affinity 50 echocardiography device (Philips Healthcare, the Netherlands) according to the recommendations of American Society of Echocardiography. Left ventricular ejection fraction (LVEF) was calculated using the modified Simpson method. Blood samples for fasting blood glucose, whole blood count, fasting lipidrofile, serum electrolytes (potassium, magnesium, calcium) and serum creatinine levels were obtained between 8:00-10:00 in the morning after at least eight hours of fasting.

\section{Electrocardiographic Evaluation}

Twelve-lead ECGs were obtained, at $20 \mathrm{~mm} / \mathrm{mV}$ amplitude and $50 \mathrm{~mm} / \mathrm{s}$ rate with standard lead positions in a supine position. Tp-e intervals were measured manually with calipers and magnifying glass to reduce the 
error rate. The QT interval was defined as the distance between the start of QRS and the last point at which the T wave intersects the isoelectric line. The TP-e interval was defined as the distance between the peak and the end of the T wave (Figure 1). TP-e interval measurement was performed using precordial surface ECG device in supine position. After normal sinus rhythm was observed by two independent cardiologists who were blinded to clinical details, the mean values of the measurements were recorded. Heart rate, RR interval and QT was evalauted by using leads and the mean results were calculated by using three consecutive cycles ${ }^{10}$. If T-wave amplitude is $<1.5$ and there is $\mathrm{U}$-wave, then that lead was excluded from the analysis. QT maximum
(QTmax) and QT minimum (QTmin) was calculated in all leads of 12-lead ECG. QT dispersion (QTd) was defined as the interval of QTmax-QTmin. Corrected QT (QTc) was calculated by using Bazzett formula ${ }^{12}$. Tp-e/ QT and Tp-e/QTc ratios were calculated based on the obtained values. The Tp-Te/QT ratio was defined as Tp-Te in lead V5 divided by QT interval in the same lead. The intra- and inter-observer variability was $4.3 \%$ and $4.9 \%$, respectively.

The study protocolwas approved by the localEthics Committee (Approval no:2017-KAEK-189-2017.08.24-12) and the study protocol was explained to each patient and the patients who gave the written consent were included in the study.

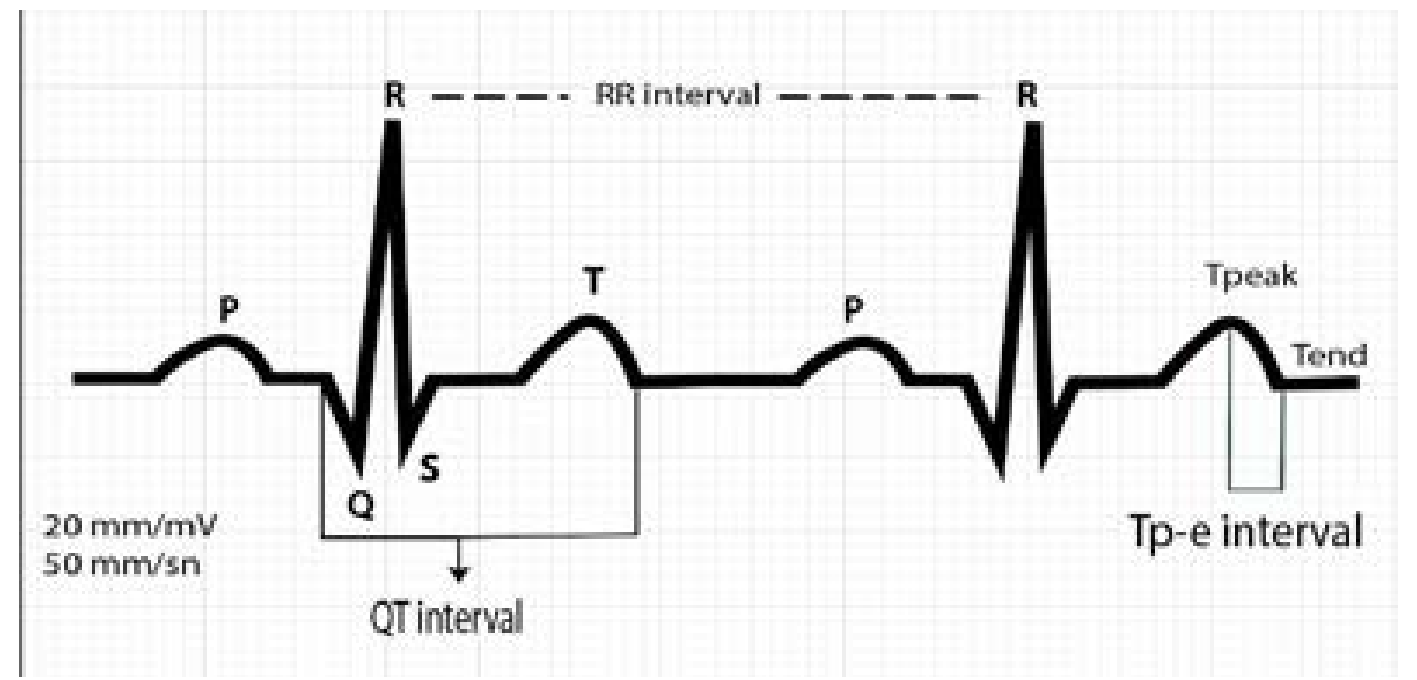

Figure 1. Demonstration of RR, QT and Tp-e intervals in electrocardiogram

\section{Statistical Analysis}

Shapiro Wilk test was used to evaluate the distribution of normality of continuous variables. Statistical analysis of clinical data between two groups was analyzed by using Student $t$ Test for normally distributed parameters and Mann Whitney $U$ test for parameters without normal distribution. For correlations, Pearson or Spearman correlation coefficient was used. Chi-square test was used for categorical variables. Statistical analyses were performed on SPSS 20 (SPSS/IBM, Chicago, IL, USA) software, and $\mathrm{p}<0.05$ was considered as the statistical significance level.

\section{Results}

The mean age of e-cigarette users was $39.1 \pm 11.4$ years and mean age of control subjects was $41.7 \pm 10.1$ years. Clinical characteristics, laboratory parameters and left ventricular ejection fraction between the two groups are summarized in Table 1 . There was no statistically significant difference between e-cigarette users and control subjects in term of sex, age, blood pressure, BMI and LVEF. The ECG characteristics of the groups, including heart rate, RR interval, Tp-e interval, corrected Tp-e interval, QTmin, QTmax, Corrected QTmin, Corrected QTmax, mean QTc, Tp-e/QT ratio, Tp-e/mean QTc ratio and QTd, are presented in Table 2. 
Table 1. Baseline characteristics and echocardiographic and laboratory parameters

\begin{tabular}{|l|l|l|l|}
\hline & $\begin{array}{l}\text { Control } \\
\text { Subjects } \\
(\mathbf{n}=\mathbf{4 0})\end{array}$ & $\begin{array}{l}\text { E-Cigarette } \\
\text { User } \\
(\mathbf{n}=\mathbf{3 6})\end{array}$ & $\begin{array}{l}\text { P- } \\
\text { value }\end{array}$ \\
\hline Age, $($ Years $)$ & $39.1 \pm 11.4$ & $41.7 \pm 10.1$ & 0.289 \\
\hline Gender, $(\mathrm{Female} / \mathrm{Male})$ & $10 / 30$ & $8 / 28$ & 0.695 \\
\hline BMI, $\left(\mathrm{kg} / \mathrm{m}^{2}\right)$ & $26.0 \pm 3.4$ & $27.3 \pm 5.8$ & 0.344 \\
\hline SBP, $(\mathrm{mmHg})$ & $114.9 \pm 9.2$ & $116.2 \pm 12.2$ & 0.534 \\
\hline DBP, $(\mathrm{mmHg})$ & $71.9 \pm 7.7$ & $72.4 \pm 9.2$ & 0.804 \\
\hline FBG, $(\mathrm{mg} / \mathrm{dL})$ & $96 \pm 12.1$ & $96.9 \pm 12.3$ & 0.447 \\
\hline Creatinine, $(\mathrm{mg} / \mathrm{dL})$ & $0.84 \pm 0.13$ & $0.84 \pm 0.14$ & 0.996 \\
\hline Total Cholesterol, $(\mathrm{mg} / \mathrm{dL})$ & $191.6 \pm 32.7$ & $191.1 \pm 32$ & 0.948 \\
\hline Triglyceride, $(\mathrm{mg} / \mathrm{dL})$ & $146.5 \pm 55.4$ & $164.4 \pm 86.7$ & 0.655 \\
\hline HDL-cholesterol, $(\mathrm{mg} / \mathrm{dL})$ & $48.3 \pm 9.7$ & $45.3 \pm 10.9$ & 0.216 \\
\hline LDL-cholesterol, $(\mathrm{mg} / \mathrm{dL})$ & $112.6 \pm 29.6$ & $110 \pm 25.9$ & 0.775 \\
\hline Hemoglobin, $(\mathrm{g} / \mathrm{dL})$ & $14.6 \pm 1.7$ & $14.4 \pm 1.3$ & 0.385 \\
\hline Leucocytes $\left.\left(10^{3} / \mathrm{mm}\right)^{3}\right)$ & $7.1 \pm 1.5$ & $6.5 \pm 1.7$ & 0.099 \\
\hline Platelets $\left(10^{3} / \mathrm{mm}^{3}\right)$ & $263.7 \pm 38.3$ & $261.3 \pm 57.7$ & 0.344 \\
\hline LVEF $(\%)$ & $61.8 \pm 2.7$ & $61.1 \pm 3$ & 0.579 \\
\hline
\end{tabular}

Expressed as Mean \pm Standard Deviation, $p$ values were compared by Student'

T-test or chi-square test as appropriate, $\mathrm{p}<0.05$ significant.

BMI: body mass index, SBP: Systolic blood pressure, DBP: Diastolic blood pressure,

FBG: Fasting blood glucose, HDL: High-density lipoprotein; LDL: Low-density

lipoprotein, LVEF: Left ventricular ejection fraction

Table 2. Electrocardiographic parameters of the study population.

\begin{tabular}{|l|l|l|l|}
\hline & $\begin{array}{l}\text { Control } \\
\text { subjects } \\
(\mathbf{n}=\mathbf{4 0})\end{array}$ & $\begin{array}{l}\text { E-Cigarette } \\
\text { User } \\
(\mathbf{n = 3 6})\end{array}$ & $\begin{array}{l}\text { P- } \\
\text { value }\end{array}$ \\
\hline Heart rate (Bpm) & $72.5 \pm 9.8$ & $76 \pm 9.1$ & $<0.001$ \\
\hline RR interval (ms) & $0.842 \pm 0.1$ & $0.806 \pm 0.2$ & 0.004 \\
\hline Tp-e (ms) & $74.9 \pm 6.4$ & $80.1 \pm 4.1$ & $<0.001$ \\
\hline Corrected Tp-e (ms) & $82.9 \pm 7.5$ & $87.8 \pm 6.3$ & 0.003 \\
\hline QT minimum (ms) & $352.8 \pm 20.6$ & $360.8 \pm 17.3$ & 0.060 \\
\hline QT maximum (ms) & $378.8 \pm 24.6$ & $388.6 \pm 16.8$ & 0.040 \\
\hline Corrected QT minimum (ms) & $389.9 \pm 20.9$ & $394.9 \pm 19.7$ & 0.280 \\
\hline Corrected QT maximum (ms) & $418.6 \pm 25.3$ & $425.1 \pm 20.4$ & 0.227 \\
\hline Mean Corrected QT (ms) & $404.3 \pm 22$ & $409.4 \pm 19.7$ & 0.291 \\
\hline QT dispersion (ms) & $25.9 \pm 13.6$ & $27.9 \pm 10.9$ & 0.183 \\
\hline Corrected QT dispersion (ms) & $28.7 \pm 14.9$ & $30.6 \pm 12.4$ & 0.268 \\
\hline Tp-e/QT (ms) & $0.20 \pm 0.013$ & $0.21 \pm 0.011$ & 0.002 \\
\hline Tpe/meanQTc (ms) & $0.18 \pm 0.016$ & $0.20 \pm 0.014$ & 0.003 \\
\hline
\end{tabular}

Expressed as Mean \pm Standard Deviation. Bpm: Beats per minute, Tp-e: Peak-to-end interval of the T wave

Baseline heart rate, RR interval was statistically significantly higher in the e-cigarette group. In the e-cigarette user group, Tp-e and Corrected Tp-e interval were significantly prolonged compared to the control subjects (80.1 \pm 4.1 milliseconds $(\mathrm{ms})$ vs. $74.9 \pm 6.4 \mathrm{~ms}, \mathrm{p}<0.001$; $87.8 \pm 6.3 \mathrm{~ms}$ vs. $82.9 \pm 7.5 \mathrm{~ms}, \mathrm{p}=0.003$, respectively). Both Tp-e/QT ratio and Tp-e/QTc ratio were found to be statistically higher in the e-cigarette users group than the healthy control group. $(0.21 \pm 0.011 \mathrm{~ms}$ vs. $20 \pm 0.013$ $\mathrm{ms}, \mathrm{p}=0.002 ; 0.20 \pm 0.014 \mathrm{~ms}$ vs. $0.18 \pm 0.016 \mathrm{~ms}, \mathrm{p}$ $=0.003$, respectively), (Table 2 ). There was no difference in terms of the other ECG parameters between the groups. E-cigarette users had been smoking for 58.2 \pm 8.4 months (range 6 to 94 months). There was no correlation between Tp-e interval, or Tpe/QT and Tpe/QTc ratio and the duration of e-cigarette use. Furthermore, there was no significant correlation between age, BMI, blood pressure with Tp-e interval, Tp-e/QT ratio, and Tp-e/QTc ratio. 


\section{Discussion}

The major new finding of this study was the determination of statistically significantly increased Tp-e interval, corrected $\mathrm{Tp}$-e and $\mathrm{Tp}$-e/QT ratio in e-cigarette users compared to healthy control subjects. These results provide important evidence on adverse effects of e-cigarette use on ventricular repolarization parameters in the acute stage. This is also the first study to report an increase in the Tp-e interval, Tp-e/QT ratio and Tp-e/ QTc ratio among e-cigarette smokers.

E-cigarettes are battery-powered devices for the dissolution of nicotine and other aromas (coffee, mint or fruity tobacco, etc.) in the liquid consisting of propylene glycol and/or vegetable glycerol and transmission of nicotine in the vapor of this liquid mixture. The most commonly used solvents for nicotine are vegetable glycerol and propylene glycol. E-liquid solution heated by the atomized in the e-cigarette cartridge creates an aerosol mimicking the cigarette smoke ${ }^{13,14}$. Other components of the solution include water, ethanol and various additives. The amounts of these substances in the cartridge vary among e-cigarette brands. Moreover, very high, high, medium, low and zero (nicotine-free) forms are available based on their nicotine content ${ }^{4}$. The nicotine content in e-cigarettes is determined by manufacturers and varies between brands and even different models of the same brand. Nicotine content in an e-cigarette liquid ranges between $0-36.6 \mathrm{mg}^{3}$. E-cigarettes have the potential to contain nicotine equal or more than conventional tobacco cigarettes ${ }^{14}$.

Ilgenli et al. included 24 long-term smokers (mean age: $40 \pm 5$ years) and 23 non-smokers (mean age: $42 \pm 10$ years) in their study where the effects of conventional smoking on ventricular repolarization were examined. Basic clinical and echocardiographic data showed no significant difference between smokers and nonsmokers. QT interval and QTc interval was similar in both groups, however, Tp-e interval $(78.9 \pm 7.3$ vs $85.3 \pm$ 10.7, $\mathrm{p}=0.02)$, Tpe-/QT ratio $(0.21 \pm 0.02$ vs $0.25 \pm$ $0.03, \mathrm{p}=0.001)$, Tpe/QTc ratio $(0.20 \pm 0.02$ vs 0.23 $\pm 0.03, \mathrm{p}=0.001)$ parameters were found to be higher in smokers in comparison to non-smokers ${ }^{15}$. In addition to these parameters (Tp-e interval and Tp-e/QT, Tp-e/QTc ratios), Tasoler et al. demonstrated increased QT and QTd values in their study ${ }^{16}$. And Kayali et al. showed that smoking had adverse effects on Tp-e interval and Tp-e/QT, Tp-e/QTc ratios in adolescents aged 16-19 years. Similar to conventional cigarette, Tp-e in- terval and Tp-e/QT, Tp-e/QTc ratios were statistically significantly higher among the e-cigarette users in our study. However, no statistically significant difference was found in terms of QTc and QTd. Based on our current knowledge, this is the first study to determine Tp-e interval and Tp-e / QT, Tp-e / QTc changes in adult e-cigarette users.

It is clearly known that nicotine released to the blood circulation during smoking increases plasma catecholamines, arterial blood pressure, and heart rate. All these changes can have an arrhythmogenic impact on heart by increasing the workload and oxygen need of myocardi$\mathrm{um}^{17}$. Moreover, e-cigarette use increases heart rate and blood pressure faster than conventional cigarettes In twenty healthy non-smoking volunteers, 10 minutes of e-cigarette containing nicotine $(18 \mathrm{mg})$ use significantly increased arterial pressure and heart rate ${ }^{18}$. In another study with a double blind design and 17 healthy subjects, the acute effects of inhaling the aerosol of e-cigarette (with and without nicotine) on vascular and pulmonary functions, nicotine e-cigarette aerosol caused a significant increase in blood pressure, heart rate and arterial stiffness ${ }^{19}$. This study demonstrates that inhaled e-cigarette aerosol with nicotine has a significant effect on vascular functions. In our findings, the heart rate was increased in the e-cigarette use group in accordance with the literature. However, arterial blood pressure increase was not consistent with the literature. This may be due to short-term resting of patients after acute e-cigarette use, and that their ECG measurements were taken first.

The duration of action potential is longer than other myocytes in medium myocardial M cells. Earliest repolarization occurs in epicardial cells. Epicardial action potential is represented with the peak of $\mathrm{T}$-wave in ECG and the end of myocardial action potential is represented with the end of the $\mathrm{T}$ wave. Therefore, $\mathrm{Tp}$-e interval is an indicator of transmural depolarization ${ }^{20,21}$. The prolongation of TP-e interval has been previously demonstrated in Brugada syndrome, long QT syndrome, hypertrophic cardiomyopathy, in conventional smokers, patients with obstructive sleep apnea and Behcet's disease ${ }^{11,15,21-23}$. Prolongation of Tp-e interval while QTc is normal has been found to be associated with sudden cardiac death ${ }^{24,25}$. In recent years, Tp-e interval and Tp-e/QT ratio have become new electrocardiographic indices of abnormal dispersion of ventricular repolarization ${ }^{26}$. Moreover, it has been claimed that these parameters can be electrocardiographic markers 
of ventricular arrhythmogenesis and sudden death ${ }^{27,28}$. Zumhagen et al. ${ }^{29}$ reported that Tp-Te/QT ratio evaluation in patients with Brugada syndrome could potentially be useful for the identification of life-threatening arrhythmias.

In most countries, the sale of nicotine-containing e-cigarettes is not permitted, but the sale of nicotine-free e-cigarettes is common and nicotine can be added to these later. When e-cigarette smoking characteristics are examined in terms of age groups, it is reported that it is preferred among young population. ${ }^{13,30}$ Our study population consisted of young adults. The fact that e-cigarette is associated with the electrocardiographic markers of ventricular arrhythmogenesis even in this group, may be considered as new evidence that e-cigarette is not harmless as perceived by users. Widespread use of e-cigarette among youth, marketing them as harmless, extensive variance among brands in terms of e-liquid content, having limited knowledge about longterm e-cigarette use, production of e-liquids in uncontrolled locations, and causing increased heart rate and ventricular repolarization parameters in a similar way to conventional cigarettes is a crucial threat in terms of public health.

\section{Limitations}

Our study has some limitations. The main limitation of our study was the relatively small number of cases and its single-center study design. Our study was cross-sectional, however, long-term prospective studies are needed to clearly demonstrate the relationship between $\mathrm{Tp}$-e interval and Tp-e/QT ratio and ventricular arrhythmia in e-cigarette users. In addition, manual measurement may have rendered the ECG results of the QT and Tp-e intervals insufficient. In high-resolution digital systems, measurements could have reduced the margin of error even further. E-cigarette usage patterns were based on declarations of users. Therefore, conventional smokers or concomitant smokers may not be excluded. Another limitation of our study was the lack of blood nicotine level testing.

\section{Conclusion}

In this cross-sectional study, important evidence demonstrating that e-cigarette use is not harmless in adults using e-cigarette is presented in comparison to healthy control participants who do not use e-cigarettes. It has been determined that e-cigarette use causes adverse alterations in ventricular repolarization, which may be potential indicators of ventricular arrhythmogenesis.

\section{Authors contribution}

Contributions: (I) Conception and design: VD, SH ; (II) Administrative support: VD, YT and HE; (III) Provision of study materials or patients: VD, HE, SH and YT; (IV) Collection and assembly of data: VD and YT; (V) Data analysis and interpretation: VD, HE and YT; (VI) Manuscript writing: All authors; (VII) Final approval of manuscript: All authors

\section{Acknowledgements}

We would like to thank all participants for participating in the study and also Abidin Işlak for the figures.

\section{Conflict of interest}

None.

\section{References}

1.Menotti A, Puddu PE, Tolonen H, Adachi H, Kafatos A and Kromhout D. Age at death of major cardiovascular diseases in 13 cohorts. The seven countries study of cardiovascular diseases 45-year follow-up. Acta Cardiol. 2019; 74 (1): 66-72.

2.Darville A and Hahn EJ. E-cigarettes and Atherosclerotic Cardiovascular Disease: What Clinicians and Researchers Need to Know. Curr Atheroscler Rep. 2019; $21(5): 15$.

3.Goniewicz ML, Gupta R, Lee YH, Reinhardt S, Kim S, Kim B et al. Nicotine levels in electronic cigarette refill solutions: A comparative analysis of products from the US, Korea, and Poland. Int J Drug Policy. 2015; 26 (6): 583-8.

4.MacDonald A, Middlekauff HR. Electronic cigarettes and cardiovascular health:what do we know so far? Vasc Health Risk Manag. 2019; 15:159-174.

5.Walsh III JA, Prineas R, Daviglus ML, Ning H, Liu $\mathrm{K}$, Lewis CE et al. Prevalence of electrocardiographic abnormalities in a middle-aged, biracial population: Coronary Artery Risk Development in Young Adults study. J Electrocardiol. 2010; 43(5):385. e1-9.

6.Algra A, Tijssen JG, Roelandt JR, Pool J, Lubsen J. QTc prolongation measured by standard 12-lead electrocardiography is an independent risk factor for sudden death due to cardiac arrest. Circulation. 1991; 83(6):1888-94

7.Lux RL. Basis and ECG measurement of global ventricular repolarization. J Electrocardiol. 2017; 50 (6):792797. 
8. Yue B, Tian $\mathrm{H}$ and $\mathrm{Xu}$ B. Research Progress in Formation and Application of Tp-Te Interval. Cardiology and Cardiovascular Research. 2019; 3 (1): 10-13.

9.Akboğa MK, Gülcihan Balcı K, Yılmaz S, Aydın S, Yayla Ç, Ertem AG, et al. Tp-e interval and Tp-e/QTc ratio as novel surrogate markers for prediction of ventricular arrhythmic events in hypertrophic cardiomyopathy. Anatol J Cardiol. 2017; 18(1):48-53

10. Castro Hevia J, Antzelevitch C, Tornés Bárzaga F, Dorantes Sánchez M, Dorticós Balea F, Zayas Molina $\mathrm{R}$, et al. Tpeak-Tend and Tpeak-Tend dispersion as risk factors for ventricular tachycardia/ventricular fibrillation in patients with the Brugada syndrome. $J$ Am Coll Cardiol. 2006; 47(9):1828-34.

11.Shimizu M, Ino H, Okeie K, Yamaguchi M, Nagata M, Hayashi K, et al. T-peak to T-end interval may be a better predictor of high-risk patients with hypertrophic cardiomyopathy associated with a cardiac troponin I mutation than QT dispersion. Clin Cardiol. 2002; 25 (7):335-339.

12.Bazett $H$. An analysis of the time-relations of electrocardiograms. Ann Noninvasive Electrocardiol. Annals of Noninvasive Electrocardiology. 1997; 2: 177-94.

13.Etter J-F. Electronic cigarettes: a survey of users. BMC Public Health. 2010; 10: 231.

14.Voos N, Goniewicz ML, Eissenberg T. What is the nicotine delivery profile of electronic cigarettes? Expert Opin Drug Deliv. 2019; 16 (11):1193-1203.

15.İlgenli TF, Tokatlı A, Akpınar O, Kılıçaslan F. The Effects of Cigarette Smoking on the Tp-e Interval, Tp-e/QT Ratio and Tp-e/QTc Ratio. Adv Clin Exp Med. 2015; 24(6):973-8.

16.Taşolar H, Ballı M, Bayramoğlu A, Otlu YÖ, Cetin M, Altun B, Cakıc1 M. Effect of smoking on Tp-e interval, Tp-e/QT and Tp-e/QTc ratios as indices of ventricular arrhythmogenesis. Heart Lung Circ. 2014; 23(9):827-32.

17.DiGiacomo SI, Jazayeri MA, Barua RS, Ambrose JA. Environmental Tobacco Smoke and Cardiovascular Disease. Int J Environ Res Public Health. 2018; 16(1). pii: E96.

18.Cooke WH, Pokhrel A, Dowling C, Fogt DL, Rickards CA. Acute inhalation of vaporized nicotine increases arterial pressure in young non-smokers: a pilot study. Clin Auton Res. 2015; 25(4):267-70.

19.Antoniewicz L, Brynedal A, Hedman L, Lundbäck
M, Bosson JA. Acute Effects of Electronic Cigarette Inhalation on the Vasculature and the Conducting Airways. Cardiovasc Toxicol. 2019; 19(5):441-450.

20. Ucar FM, Ozturk C, Yllmaztepe MA. Evaluation of Tp-e interval, Tp-e/QT ratio and Tp-e/QTc ratio in patients with acute myocarditis. BMC Cardiovasc Disord. 2019; 19(1):232.

21.Yan GX, Antzelevitch C. Cellular basis for the normal $\mathrm{T}$ wave and the electrocardiographic manifestations of the long-QT syndrome. Circulation. 1998; 98(18):1928-36.

22.Topilski I, Rogowski O, Rosso R, Justo D, Copperman Y, Glikson M, et al. The morphology of the QT interval predicts torsade de pointes during acquired bradyarrhythmias. J Am Coll Cardiol. 2007; 49(3):320-8. 23.Hidayet Ş, Demir V, Turan Y, Gürel G, Taşolar MH. Evaluation of Tp-e interval, Tp-e/QT ratio, and Tp-e/ QTc ratio in patients with Behçet's disease. Anatol J Cardiol. 2019;22(2):85-90.

24.Wellens HJ, Schwartz PJ, Lindemans FW, Buxton AE, Goldberger JJ, et al. Risk stratification for sudden cardiac death: current status and challenges for the future. Eur Heart J. 2014; 35(25):1642-51.

25. Panikkath R, Reinier K, Uy-Evanado A, Teodorescu C, Hattenhauer J, Mariani R, et al. Prolonged Tpeakto-tend interval on the resting ECG is associated with increased risk of sudden cardiac death. Circ Arrbythm Electrophysiol. 2011;4(4):441-7.

26.Gupta P, Patel C, Patel H, Narayanaswamy S, Malhotra B, Green JT, et al. T(p-e)/QT ratio as an index of arrhythmogenesis. J Electrocardiol. 2008; 41(6):567-74.

27. Kors JA, Ritsema van Eck HJ, van Herpen G. The meaning of the Tp-Te interval and its diagnostic value. J Electrocardiol. 2008;41(6):575-80.

28. Dilaveris P, Pantazis A, Gialafos E, Triposkiadis F, Gialafos J. The effects of cigarette smoking on the heterogeneity of ventricular repolarization. Am Heart J. 2001;142(5):833-7.

29. Zumhagen S, Zeidler EM, Stallmeyer B, Ernsting M, Eckardt L, Schulze-Bahr E. Tpeak-Tend interval and Tpeak-Tend/QT ratio in patients with Brugada syndrome. Europace. 2016;18(12):1866-1872.

30. Yan XS, D'Ruiz C. Effects of using electronic cigarettes on nicotine delivery and cardiovascular function in comparison with regular cigarettes. Regul Toxicol Pharmacol. 2015;71(1):24-34. 